FACTA UNIVERSITATIS

Series: Mechanical Engineering Vol. 17, No 1, 2019, pp. 87 - 93

https://doi.org/10.22190/FUME190103012L

Short communication

\title{
NUMERICAL IMPLEMENTATION OF FRETTING WEAR IN THE FRAMEWORK OF THE MDR
}

\author{
Qiang Li, Fabian Forsbach, Justus Benad \\ Berlin University of Technology, Berlin, Germany
}

\begin{abstract}
Two numerical methods are proposed to improve accuracy of the numerical calculation of fretting wear in the framework of the Method of Dimensionality Reduction $(M D R)$. Due to the singularity of the transformation equations, instabilities appear at the border between the stick and slip regions after many transformations from the one-dimensional to the three-dimensional contact and back. In these two methods, the transformation equations are reformulated to weaken the singularity of the integrals and a stable simulation of fretting wear is realized even with the wear models which go beyond the classical Archard law. With an example of dual-oscillation, we show the change in the worn profile of a parabolic indenter as well as the stress distribution on the contacting surface during the oscillating cycles under the Archard's law of wear and Coulomb's law of friction.
\end{abstract}

Key Words: Fretting Wear, Method of the Dimensionality Reduction, Singularity, Numerical Simulation

\section{INTRODUCTION}

In recent years, the Method of the Dimensionality Reduction has been applied to various contact problems. The classic contacts of rotationally symmetric indenters like the Hertzian normal contact, partial sliding, or JKR (Johnson-Kendall-Roberts)-type adhesive contact, etc. can be easily understood and resolved very quickly in the framework of the MDR [1]. A similar approach, the Method of Memory Diagrams (MMD), provides semi-analytical solutions for axisymmetric contact problems, for example, friction-induced energy loss [2]. The paper [3] provides a series of guidelines for using the MDR in the applications of homogeneous media or graded material, in elastic and viscoelastic contacts. If the contacting bodies and the material properties do not change in the whole contact case, one can obtain the results by simulation merely in the framework of the one-dimensional contact, for example, relaxation damping [4]

Received January 03, 2019 / Accepted March 01, 2019

Corresponding author: Qiang, $\mathrm{Li}$

Affiliation: Berlin University of Technology, Sekr. C8-4, Straße des 17. Juni 135, D-10623 Berlin

E-mail: qiang.li@tu-berlin.de 
or adhesive pull-off [1]. However, in some cases, for example, in the wear contact, the surface profile of the indenter changes due to wear so that one has to come back to the three-dimensional contact by using the transformation equations to calculate a new surface profile. Then the 3D profile is transformed into the corresponding new 1D profile for the solution of the contact problem. The procedure is then repeated for the whole contact. Fig.1 shows this simulation procedure as presented in the papers [5, 6].

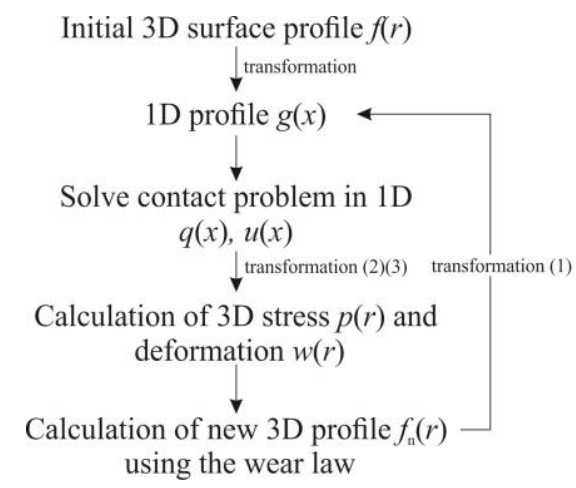

Fig. 1 Procedure of numerical calculation of wear using the MDR

These transformation equations are listed in the following for the profile, from 3D $f(r)$ to $1 \mathrm{D}$ $g(x)$, and for the displacement and stress, from 1D $u(x), q(x)$ to 3D $w(r), p(r)$ :

$$
\begin{aligned}
& g(x)=|x| \int_{0}^{|x|} \frac{f^{\prime}(r)}{\sqrt{x^{2}-r^{2}}} \mathrm{~d} r, \\
& w(r)=\frac{2}{\pi} \int_{0}^{r} \frac{u(x)}{\sqrt{r^{2}-x^{2}}} \mathrm{~d} x, \\
& p(r)=-\frac{1}{\pi} \int_{r}^{\infty} \frac{q^{\prime}(x)}{\sqrt{x^{2}-r^{2}}} \mathrm{~d} x .
\end{aligned}
$$

These Abel equations have a singularity at point $x=r$. Numerically, the integral can be calculated in different ways, for example, using Simpson's $1 / 3^{\text {rd }}$ rule, or via a semianalytical technique with piecewise approximation in a segment by a constant or linear profile $[7,8]$. From Fig.1, we can see that in the case of numerical description of wear, the transformation from $1 \mathrm{D}$ to $3 \mathrm{D}$ and the back transformation have to be carried many times, which will lead to instability if the integral is numerically approximated with a low accuracy. In the recent paper [9], the transformations (1)-(3) are rewritten by using the integration by parts to avoid the singularity, and the corresponding numerical implementation is given in detail. With an example of gross slip wear, this method shows very accurate results, where, however, only transformations (1) and (3) are necessary. In the fretting wear, we observe instability using the same method where the Eq. (2) is also involved. In this short communication, we will give two further approaches to improve the integral precision and stability in the fretting wear simulation. 


\section{METHODS}

\subsection{Method A}

As suggested in [10], the singularity of the integrand can be weakened by splitting it into two parts to improve accuracy of an approximate integration. Focusing on the Eq. (2), it can be rewritten as

$$
w(r)=\frac{2}{\pi} \int_{0}^{r}\left(\frac{u_{1}(x)}{\sqrt{r^{2}-x^{2}}}+\frac{u_{2}(x)}{\sqrt{r^{2}-x^{2}}}\right) \mathrm{d} x
$$

with

$$
u_{1}(x)=x u^{\prime}(x), u_{2}(x)=u(x)-x u^{\prime}(x) .
$$

Eq. (4) can further be written with a derivative:

$$
w(r)=\frac{2}{\pi} \int_{0}^{r}\left(u^{\prime}(x) \frac{-\mathrm{d} \sqrt{r^{2}-x^{2}}}{\mathrm{~d} x}+\left[u(x)-x \cdot u^{\prime}(x)\right] \cdot \frac{\mathrm{d} \sin ^{-1}(x / r)}{\mathrm{d} x}\right) \mathrm{d} x .
$$

This integral can be numerically calculated with greater accuracy than Eq. (2). In a discrete form, Eq. (6) is

$$
\left.w_{j}=\frac{2}{\pi} \sum_{k=1}^{j-1}\left[\begin{array}{l}
u_{k}^{\prime}\left(-\sqrt{r_{j}^{2}-x_{k+1}^{2}}+\sqrt{r_{j}^{2}-x_{k}^{2}}\right) \\
+\left(u_{k}-u_{k}^{\prime} \cdot x_{k}\right) \cdot\left(\sin ^{-1}\left(\frac{x_{k+1}}{r_{j}}\right)-\sin ^{-1}\left(\frac{x_{k}}{r_{j}}\right)\right)
\end{array}\right)\right] .
$$

First derivative $u_{k}^{\prime}$ is obtained via forward difference.

For Eqs. (1) and (3), however, we use the method from the recent paper [9] which gives highly accurate results.

\subsection{Method B}

Here we rewrite all the three transformations (1)-(3) simply in the following form

$$
\begin{gathered}
g(x)=|x| \int_{0}^{|x|} f^{\prime}(r) \cdot \frac{\mathrm{d} \sin ^{-1}(r / x)}{\mathrm{d} r} \mathrm{~d} r, \\
w(r)=\frac{2}{\pi} \int_{0}^{r} u(x) \cdot \frac{\mathrm{d} \sin ^{-1}(x / r)}{\mathrm{d} x} \mathrm{~d} x, \\
p(r)=-\frac{1}{\pi} \int_{r}^{\infty} q^{\prime}(x) \cdot \frac{\mathrm{d} \cosh ^{-1}(x / r)}{\mathrm{d} x} \mathrm{~d} x .
\end{gathered}
$$

Using the trapezoidal method, they can be written in the discrete form

$$
g_{j}=x_{k} \sum_{k=2}^{k=j} \frac{f_{k-1}^{\prime}+f_{k}^{\prime}}{2} \cdot\left[\sin ^{-1}\left(r_{k} / x_{j}\right)-\sin ^{-1}\left(r_{k-1} / x_{j}\right)\right],
$$




$$
\begin{gathered}
w_{j}=\frac{2}{\pi} \sum_{k=2}^{k=j} \frac{u_{k-1}+u_{k}}{2} \cdot\left[\sin ^{-1}\left(x_{k} / r_{j}\right)-\sin ^{-1}\left(x_{k-1} / r_{j}\right)\right], \\
p_{j}=-\frac{1}{\pi} \sum_{k=j+1}^{k=N} \frac{q_{k-1}^{\prime}+q_{k}^{\prime}}{2} \cdot\left[\cosh ^{-1}\left(x_{k} / r_{j}\right)-\cosh ^{-1}\left(x_{k-1} / r_{j}\right)\right] .
\end{gathered}
$$

The first derivatives in Eqs. (11)-(13) can be obtained via central differences. Different from the method in [9] by using the technique of integration by parts, the second derivatives are not necessary in the above Method B.

Fig. 2 shows the numerical errors of 1D profile, normal stress and deformation at each discrete point in the contact area in comparison with the Hertzian theory. The error is estimated as the absolute value of (Results A or B - Theory)/Theory. It is noted that the adjustment as suggested in [9] is not applied for stress in the method A. It is seen that for the profile, there is no difference for the two methods, but for the deformation, the method $\mathrm{A}$ is better.

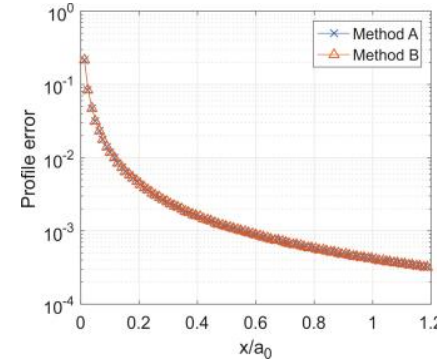

a)

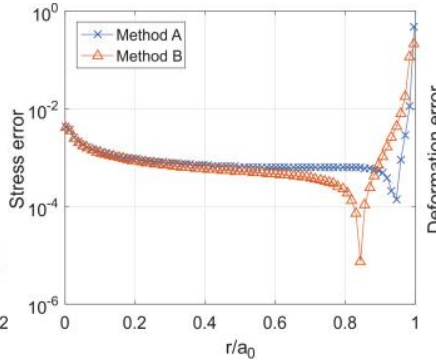

b)

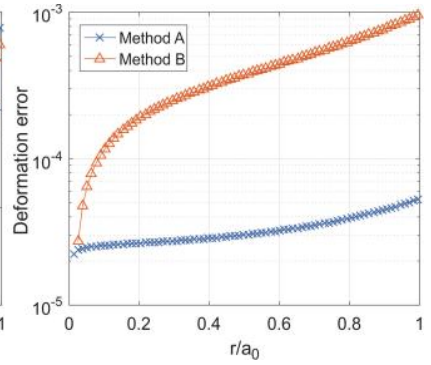

c)

Fig. 2 Error estimation with an example of Hertzian contact for: (a) 1D profile; (b) normal stress; (c) normal deformation

\section{IMPLEMENTATION OF FRETTING WEAR}

Now we numerically simulate fretting wear with the approaches given in Section 2 . The indenter with initial parabolic profile $f=r^{2} /(2 R)$ is pressed into the elastic half space with elastic modulus $E^{*}$ and Poisson's ratio $v$. The indenter oscillates with displacement-controlled periodic functions in both vertical and horizontal directions:

$$
\begin{aligned}
& u_{z}(t)=u_{z}^{(0)}+\Delta u_{z} \sin \omega_{1} t \\
& u_{x}(t)=u_{x}^{(0)}+\Delta u_{x} \sin \left(\omega_{2} t+\varphi\right)
\end{aligned} .
$$

The numerical algorithm is the same as in Fig.1, and we use the local formulation of Archard's law of wear for the change in 3D surface profile:

$$
\Delta f(r)=k_{\text {wear }} \tau(r)\left(\Delta u_{x}^{(0)}-\Delta u_{x, 3 \mathrm{D}}(r)\right),
$$

where $k_{\text {wear }}$ is the wear coefficient, $\tau(r)$ is the tangential stress on the surface, $\Delta u_{x}{ }^{(0)}$ is the tangential movement of the indenter and $\Delta u_{x, 3 \mathrm{D}}$ is the change of tangential displacement of the elastic half space in a time increment. 


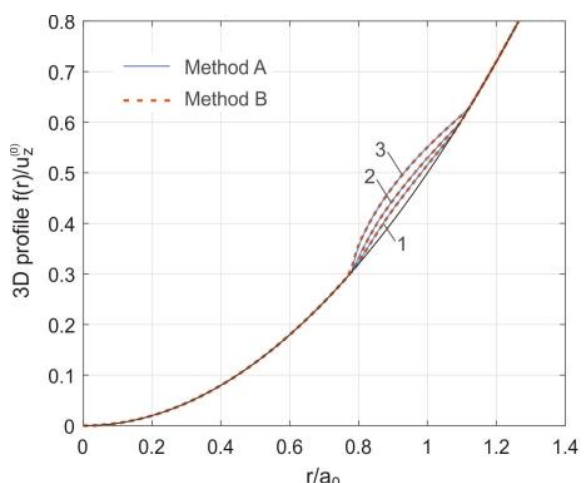

a)

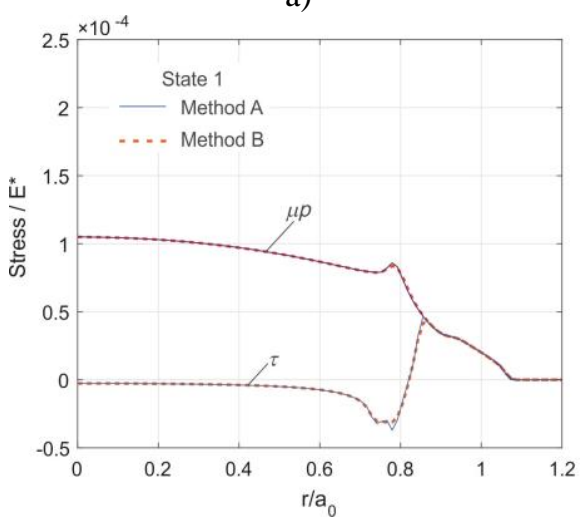

c)

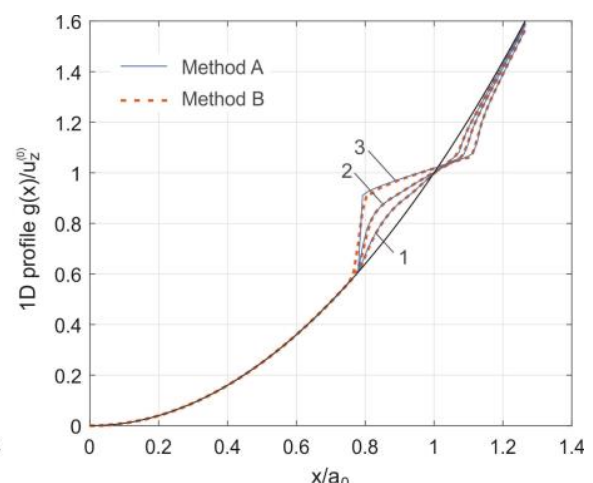

b)

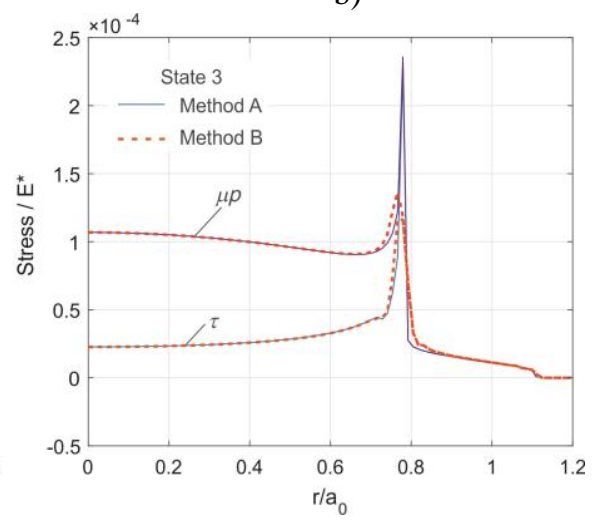

d)

Fig. 3 Simulation of fretting wear: (a) 3D profiles and (b) corresponding 1D profiles at three states; (c) normal and tangential stresses at state 1 and (d) state 3

It is known that if the tangential oscillation amplitude is small, there will be a stick region in the center and a slip region at the boundary of the contact area with Coulomb's law of friction. The contact problem can be solved in the 1D contact with Winkler's spring foundation [3]. The obtained 1D tangential stress $q_{x}$ and tangential movement $u_{x, 1 \mathrm{D}}$ are then transformed into three-dimension $\tau(r)$ and $u_{x, 3 \mathrm{D}}$ for substitution into the wear law (5) to get the new profile. In the MDR, the stick and slip regions of 'springs' are determined according to the following rule:

$$
\begin{array}{ll}
\Delta u_{x}(x)=\Delta u_{x}^{(0)}, & \text { in the stick region, if }\left|k_{x} u_{x}(x)\right|<\mu f_{z}(x) \\
u_{x}(x)= \pm \frac{\mu f_{z}(x)}{k_{x}}, & \text { in the slip region }
\end{array} .
$$

In the numerical simulation, we first assume that all the springs in the contact region move with the same value $\Delta u_{x}{ }^{(0)}$ as the indenter and then the tangential forces of these springs are calculated $k_{x} u_{x, \text { pre }}$. Subsequently, we check the condition (15): the springs that meet condition $k_{x} u_{x, \text { pre }}\left\langle\mu f_{z}\right.$ are located in the stick region, where the relative tangential movement is zero, $\Delta u_{x}=\Delta u_{x}{ }^{(0)}$, as described in (15). The other springs having the relation 
$k_{x} u_{x, p r e}>\mu f_{z}$ are in the slip region, where the tangential displacement must be smaller than assumed value $u_{x}<u_{x, p r e}$, and it will be calculated with the rule in (15). In fretting wear, the indenter moves also backward, so the sign in (15) should be the same as $u_{x, p r e .}$

Numerically, we simulated various cases of the fretting, including variation of phase, different oscillation frequencies in normal and tangential directions, amplitude of oscillation, coefficient of friction, and also other laws of wear, for example the case when the surface change has a power-law function of stress or relative tangential movement. Both methods show stable results. Here we give only one example as shown in Fig.3. The main parameters are set as $\Delta u_{x}{ }^{(0)}=\Delta u_{z}{ }^{(0)}=0.1 u_{z}{ }^{(0)}, u_{x}{ }^{(0)}=0, \varphi=\pi / 4, \omega_{1}=\omega_{2}=100, \mu=0.1$ and discrete points $N=100$. The states $1,2,3$ in Figs.3a and b indicate 50.72, 100 and 199.24 cycles of oscillation (the large value of wear coefficient is used to observe the wear behavior quickly). The 3D worn profile and the corresponding 1D profile are shown in Figs.3a and b. Figs.3c and d show the 3D normal and tangential stresses at state 1 and 3, where the indenter is located shortly before $3 / 4$ and $1 / 4$ period, respectively. The difference of the $3 \mathrm{D}$ profile between these two methods is very small. Only when the surface is strongly worn, the stress singularity at the border of stick region can be observed.

\section{CONCLUSION}

We provide two approaches to improving the accuracy of the numerical implementation of the transformations for the fretting wear. Both the methods provide a fast and stable simulation in the fretting contact. Besides the example presented in Section 4, we have tried a few simulations with other laws of wear, for example $\Delta f \sim \tau^{1.5}$, and using not only the Methods A and $\mathrm{B}$ but also their combinations. It is found that Method B gives sometimes unstable simulations for a relatively large wear coefficient. Generally, for the wear or other contact problems, we suggest the users of the MDR to use the numerical implementation of transformations in the following consequents: a) Method A; b) combination of Method A for Eqs. (1) and (3) and Method B for Eq. (2); c) Method B. In the tests there is almost no difference observed between suggestions (a) and (b).

Acknowledgements: The authors thank Dimaki A.V. for providing the numerical code for fretting contact studied in [5] and thank Popov V.L. for the discussion.

\section{REFERENCES}

1. Popov, V.L., Heß, M., 2015, Method of dimensionality reduction in contact mechanics and friction, Springer, Berlin.

2. Kevin Truyaert, K., Aleshin, V., Koen Van Den Abeele, Delrue, S., 2019, Theoretical calculation of the instantaneous friction-induced energy losses in arbitrarily excited axisymmetric mechanical contact systems, Int. J. Solids Struct., 158, pp. 268-276.

3. Popov, V.L., Heß, M., 2014, Method of dimensionality reduction in contact mechanics and friction: a user's handbook. I. Axially-symmetric contacts, Facta Universitatis-Series Mechanical Engineering, 12, pp. 1-14.

4. Popov, M., Popov, V.L., Pohrt, R., 2015, Relaxation damping in oscillating contacts, Sci. R., 5, 16189

5. Dimaki, A.V., Dmitriev, A.I., Chai, Y.S., Popov, V.L., 2014, Rapid Simulation Procedure for Fretting Wear on the basis of the method of dimensionality reduction, Int. J. Solids Struct., 51, pp. 4215-4220. 
6. Dimaki, A.V., Dmitriev, A.I., Menga, N., Papangelo, A., Ciavarella, M., Popov, V.L., 2016, Fast High-Resolution Simulation of the Gross Slip Wear of Axially Symmetric Contacts, Tribol. Trans., 59, pp. 189-194.

7. Dasch, C.J., 1992, One-dimensional tomography: a comparison of Abel, onion-peeling, and filtered backprojection methods, Appl. Opt., 31, pp. 1146-1152.

8. Kolhe, P.S., Agrawal, A.K., 2009, Abel inversion of deflectometric data: comparison of accuracy and noise propagation of existing techniques, Appl. Opt., 48, pp. 3894-3902.

9. Benad, J., 2018, Fast numerical implementation of the MDR transformations, Facta Universitatis-Series Mechanical Engineering, 16, pp. 127-138.

10. Krylov, V.I., 1962, Approximate calculation of integrals, The Macmillan Company, Now York. 